\title{
Relocations and transfers of dying persons in hospitals and nursing homes
}

\author{
Wolfgang Michael George 1* $^{*}$
}

George WM. Relocations and transfers of dying persons in hospitals and nursing homes. Curr Res Integr Med 2017;2(1):58.

An exploratory study in over 160 hospitals and nursing homes in Germany shows that approximately 20\% (that means about 150.000) of all dying patients are

In 2015 about $80 \%$ of all Germans died in hospitals and nursing homes (1). This shows the outstanding importance of inpatient care in end of life caring. A first, own study in 2014 provided indications that the patient's last phase of life could lead to relocation (2). And this, although it is the goal of all caregivers to recognize the dying as such (3), and to allow a dignity of death independently of the place of dying (4), because relocation basically means risks and stress for all concerned (5).

\section{METHODS}

In order to overcome this information deficit, a 27-question-wide questionnaire was developed and addressed to employees from hospitals and nursing homes in 2016. The questionnaire determines the frequency of relocation (a), the date (b), the address of the relocation (c) and the influencing factors which justify or moderate the transfers (d). The questionnaire was answered particularly by nurses and doctors. The questionnaire could be answered in writing and in an online procedure. As far as possible and meaningful, the data of the total of 545 interviewees were clustered so that each institution was included as an average case in the statistical evaluation (6). In this way, 101 hospitals and 65 nursing homes of different sizes, carrier companies and federal states were included in the statistical evaluation. The study has an exploratory character. The results should be reviewed by available supply data and further studies should be supplemented.

\section{RESULTS}

The following central findings were found: In approximately $20 \%$ of the hospitals and approx. $25 \%$ of the inpatient nursing homes there are transfers of dying people. This would mean a total of approx. 150,000 patients. Nursing homes transfer dying patients primarily to hospitals and only in very few cases in a hospice or home. Hospitals' employees state that most of their transfers are carried out within their own hospital. In some cases, the relocation to a hospice takes place in a few homes. In the study, no clear preference of the day of the week or of the time could be identified. Physiological impairments such as dyspnoea, painful conditions or the deterioration of the general condition, can be identified as the central reasons for relocations. In contrast, mental reasons such as anxiety, confusion, or depressive mood are less important. Both the achieved quality of the family integration as well as the cooperation with the family doctor have an impact on a possible relocation behavior of the institutions. The better they succeed, the lower the relocation. Written patient interventions have a medium to distinct influence. The relocation behavior in the hospitals differs from that of the nursing homes. The nursing facilities are much less prepared for physical deterioration. On the other hand, psychological or mental challenges in nursing homes are better managed. The influence of the family doctor, the relatives and the patient is also lower in the hospitals. Basically, existing personal shortcomings are regarded as more problematic relocated during their last days of life. The following main risks were identified: acute physical and mental deterioration and the external influence of the physicians or relatives. The surveyed employees show ways to improve the situation.

Key Words: End-of-life care, Relocation of dying patients

for the relocation behavior, as acute personal shortcomings. This assessment unites the employees.

In open questions, respondents were given the opportunity to provide information on how to avoid relocation. The following recommendations were formulated: forward looking planning (a) to further develop home care(b), to improve cooperation with hospices and specialized palliative services (c), to improve the medical attitude (d), to increase the number and efficacy of patient care (e ), better medical education $(\mathrm{f}$ ), prevention of inappropriate use of intensive care (g), better and earlier counseling of relatives and patients (h), adaptation of premises (i), binding establishment of ethical teams (j).

\section{DISCUSSION}

For nursing homes, they are even better placed to deal with the deterioration of their patients, so that dying people are not identified as "medical emergencies", with all the problematic consequences (7). In the hospitals is quite similar. Here, too, the general units of care must be supported in such a way that dying persons are not yet moved into monitoring units as ICM or IMC. It is hoped that with the introduction of a new law (HPG) 2015, the situation for dying people in the hospitals and nursing homes in Germany will continue to improve. This includes less relocations of dying patients.

\section{REFERENCES}

1. Gesundheit, Diagnosedaten der Patienten- und Patientinnen in Krankenhäusern (einschließlich Sterbefälle), Statistisches Bundesamt Wiesbaden, 2016.

2. George WM. Place of death nursing home, MMW Fortschritte der Medizin, Originalien. 2015; II/15:17-25.

3. Bailey FA, Harman SM, Bruera E, et al. Palliative care: The last hours and days of life. Up to date, Bruera, E, Arnold, RM (Ed) Walham, MA. 2014

4. Kinoshita H, Maeda I, Morita T, et al. Place of death and the differences in patient quality of death and dying and caregiver burden. Journal of Clinical Oncology, 2015;33(4):357-63.

5. Uhrenfeldt L, Aagaard H, Hall EO, et al., A qualitative meta-synthesis of patients' experiences of intra and inter hospital transitions. Journal of Advanced Nursing, 2013;69(8): 1678-90.

6. Bortz, J. Lehrbuch der Statistik: Für Sozialwissenschaftler. SpringerVerlag. 2013

7. George WM. New seal of quality as a result of empirical studies: Palliativefriendly Hospital and Palliative-friendly Residential Care Facility, Journal of Palliative Care \& Medicine. 2016;6:2.

TransMIT Project Division for Health Services Research and Consulting, THM-University of Applied Science, Germany

Correspondence: Wolfgang Michael George, PhD Psychologist, Jahnstraße, 14 D-35394 Giessen. Telephone: 0049-641-9482111, E-mail: George@transmit.de Received: January 07, 2017, Accepted: April 15, 2017, Published: April 22, 2017 